\title{
Clinical Dimensions of Bloating in Functional Gastrointestinal Disorders
}

\author{
Min Sun Ryu, ${ }^{1}$ Hye-Kyung Jung, ${ }^{1 *}$ Jae-in Ryu, ${ }^{1}$ Jung-Sook Kim, ${ }^{2}$ and Kyung Ae Kong ${ }^{3}$ \\ ${ }^{I}$ Department of Internal Medicine, Ewha Womans University School of Medicine, Seoul, Korea; ${ }^{2}$ Health Promotion Center, Ewha Womans \\ University Medical Center, Seoul, Korea; and ${ }^{3}$ Clinical Trial Center, Ewha Womans University Medical Center, Seoul, Korea
}

\section{Background/Aims}

Bloating is common bothersome symptoms and most studies conducted in the Western countries found that bloating was frequently associated with lower gastrointestinal (GI) symptoms but many patients complaint bloating as upper Gl symptoms in the clinical setting. This study was conducted to assess the prevalence of bloating, and to identify symptom grouping and finally document the impact of bloating in the diagnosis of functional Gl disorders.

\section{Methods}

Participants in a comprehensive health-screening cohort were enrolled. They were asked about demographic, medical, and social history and upper and lower GI symptoms by using a validated questionnaire. Factor analysis with principal component analysis method with varimax rotation was used.

\section{Results}

Among the total of 1050 subjects (mean age, $44.6 \pm 10.2$ years; females, 46.4\%), significant bloating symptoms were found in 282 (26.9\%); the prevalence of functional bloating was 6.9\%. Factor analysis revealed a 5-component structure with upper Gl symptoms, constipation, diarrhea-predominant irritable bowel syndrome (IBS), constipation-predominant IBS, and fecal incontinence. Abdominal bloating loaded on both the upper GI symptoms ( 0.51 of loadings) and constipation (0.40). On logistic regression analysis, bloating was more predictable for IBS $(O R, 7.5 ; P<0.001)$ than functional dyspepsia (FD; $O R, 3.7 ; P=0.002)$. Bloating was more frequently combined with IBS according to their severity, but this association was not detected in patients with FD.

\section{Conclusions}

Abdominal bloating is common symptom in about a quarter of patients and appears as upper as well as lower $\mathrm{Gl}$ symptoms. However, abdominal bloating is more predictable for IBS, especially constipation-predominant IBS, than FD.

(J Neurogastroenterol Motil 2016;22:509-516)

\section{Key Words}

Bloating; Dyspepsia; Epidemiology; Irritable bowel syndrome

Received: October 13, 2015 Revised: December 28, 2015 Accepted: January 17, 2016

(a) This is an Open Access article distributed under the terms of the Creative Commons Attribution Non-Commercial License (http://creativecommons. org/licenses/by-nc/4.0) which permits unrestricted non-commercial use, distribution, and reproduction in any medium, provided the original work is properly cited.

*Correspondence: Hye-Kyung Jung, MD Department of Internal Medicine, Ewha Womans University Medical Center, 1071, Anyangcheon-ro, Yangcheon-gu, Seoul 07985, Korea

Tel: +82-2-2650-2874, Fax: +82-2-2655-2874, E-mail: junghk@ewha.ac.kr 


\section{Introduction}

Abdominal bloating is a common symptom affecting up to $76 \%$ of patients with functional gastrointestinal disorders (FGIDs) and $6-13 \%$ of the general population in Western countries. ${ }^{1,2}$ Regardless of the symptoms experienced, the subjective interpretation of a particular symptom as most bothersome or unpleasant is likely to determine the behavioral response of the patient. Evidence suggests that abdominal pain is the predominant symptom of patients with irritable bowel syndrome (IBS), and highly correlates with gastrointestinal (GI) symptom severity and the presence of psychiatric comorbidity. ${ }^{3-5}$ However, a previous study reported that more than $75 \%$ of patients with bloating and/or visible distension rated their symptoms as moderate to severe, and $54 \%$ reported that these symptoms affected their daily activities, while $43 \%$ took medications. ${ }^{6}$ Physicians may perceive bloating as bothersome and annoying but not life threatening; therefore, bloating is less frequently measured despite its prevalence and importance.

The few studies that adequately separated bloating from IBS and other FGIDs showed that bloating was listed by $60 \%$ of patients with IBS as the most bothersome symptom, whereas only $29 \%$ rated abdominal pain as their most bothersome symptom. ${ }^{7}$ In this study, abdominal pain as the predominant symptom of IBS was related to rectal hypersensitivity to repetitive colonic distensions; however, other non-painful IBS symptoms, such as bloating, were not, although most clinical and psychological parameters were similar. Although abdominal bloating is frequently accompanied by IBS, bloating might be a heterogeneous condition in which the pathophysiological mechanisms differ from those of IBS.

The majority of research on bloating has involved subjects in Western countries who have other FGIDs in addition. Up to $76 \%$ of IBS patients report bloating as a symptom. ${ }^{7-9}$ Few studies on abdominal bloating have been conducted in Asia. ${ }^{8,9}$ In Malaysia, dyspepsia was reported in up to $15 \%$ of a rural and $25 \%$ of an urban population, and "a lot of wind" and bloating in the "stomach" might be the most-bothersome dyspeptic symptoms. ${ }^{10}$ A factor analysis of dyspeptic symptoms in the European general population revealed that the symptom of bloating was grouped with postprandial distress syndrome, a subtype of functional dyspepsia (FD). ${ }^{11}$ In a physiologic study of patients with FD, over $80 \%$ of dyspeptic patients experienced bloating symptoms, but the proportion experiencing bloating was not different according to impaired gastric accommodation or delayed gastric emptying. ${ }^{12}$

Expert consensus has proposed to define dyspepsia as pain or discomfort in the upper abdomen, but differentiation of FD pain from IBS pain by both patients and physicians is difficult. ${ }^{11}$ However, most existing studies involving factor analysis were performed to group the GI symptoms in patients with upper GI symptoms only or lower GI symptoms only. ${ }^{2-4,10,11}$ Comprehensive study of the clinical profiles of abdominal bloating will assist selection of more focused FGIDs and specific targeted therapy.

We undertook this survey using a validated questionnaire including both upper and lower GI symptoms in a health-screening population, a representative sample of the general population. The aims of this study were to identify the prevalence of abdominal bloating with and without overlapping FGIDs, to evaluate the symptom grouping by factor analysis, and finally to document the role of abdominal bloating in the diagnosis of FGIDs.

\section{Materials and Methods}

\section{Subjects}

The study subjects consisted of 1149 who received medical check-up at Ewha Womans University Health Promotion Center. Subjects who were older than 19 years old and agreed to participate to the study were included. Subjects with severe chronic diseases, life-threatening illness or serious mental disorder belong to exclusion criteria, but these patients were not found. The patients who were diagnosed gastric cancer $(n=8)$, gastric and duodenal ulcer $(n=38)$ or reflux esophagitis $(n=36)$ and who underwent gastrectomy $(n=17)$ were also excluded. There were no subjects who had diverticulitis, inflammatory bowel disease or colon cancer. This study was approved by the Ethics Committee of the Ewha Womans University Mokdong Hospital.

\section{Questionnaires}

Subjects were given the list of GI symptoms by validated Korean version of the bowel disease questionnaire (K-BDQ) following the Rome III criteria and were instructed to check symptoms. The $\mathrm{K}-\mathrm{BDQ}$ is a tool for distinguishing several functionally diverse bowel diseases, such as IBS, FD, functional bloating, functional constipation, and functional diarrhea. Intelligibility of the questionnaire was maximized by adhering to an assumed sixth grade reading level. A previous study showed the K-BDQ to be reliable, with a median kappa statistic for symptom items of 0.74 (range, 0.36-1.00). ${ }^{13}$ Also, questions were designed to evaluate bowel habit dysfunction and to measure the degree of somatization. Severe somatization was defined as somatization symptom scores $\geq 1.0$. 
Information regarding the covariates included demographics, body mass index (BMI), co-morbidities, such as diabetes mellitus or hypertension, alcohol consumption, smoking habits, exercise, and history of cholecystectomy; hypertension and diabetes mellitus were defined as having medical history of hypertension or diabetes diagnosis by physicians. Depression was defined by having medical history of depression or having self-reported depressed mood at least once per week. The total amount of alcohol consumed per week was calculated in grams. An alcohol user was defined as someone who drank $\geq 210 \mathrm{~g} /$ week alcohol for males and $\geq 140 \mathrm{~g} /$ week for females. Smoking status was categorized into current and noncurrent smokers. Regular exercise was defined as exercise at least 3 times per week, for at least 30 minutes per session. Obesity was defined as a $\mathrm{BMI}>25 \mathrm{~kg} / \mathrm{m}^{2}$.

All FGIDs were diagnosed using Rome III criteria. ${ }^{14}$ Severity of IBS was graded into 3 classes according to frequency of abdominal pain: severe, more than once a day; moderate, 2-6 times per week; and mild, 2-4 times per months. In FD, the severity was defined by composite score of early satiety, postprandial fullness, and epigastric pain or soreness: severe, $>4$; moderate, $2-3$; and mild, 1 .

\section{Statistical Methods}

Continuous variables were expressed as mean \pm standard deviations (SDs). Univariate associations were analyzed using Student's $t$ test for continuous variables and Pearson's Chi-squared test and Fisher's exact test for categorical variables. Somatic symptom checklist score was calculated as the mean of the frequency and severity scores for each item. Somatic symptom checklist score $\geq 1$ was defined as severe. The odds ratios (ORs) and corresponding $95 \%$ confidence intervals (CIs) were computed from the coefficients in the logistic regression models.

Factor analysis (principal component analysis) was applied to assess the presence of correlations between particular types of upper or lower GI symptoms in the patients. The variables included the 19 GI symptoms coded either in the presence (1-6) or absence (0) of any intensity. The principal components analysis method of data extraction was used. An eigenvalue $>1$ (Kaiser criterion) was used to determine the number of components needed to represent the study data. A scree plot of eigenvalues was computed. To improve interpretation of the components, they were rotated using the varimax technique. Only loadings of 0.4 or higher were considered in the interpretation of factors.

Logistic analysis was performed to find the risk factors for abdominal bloating. To assess the association of bloating and FGIDs, 3 models of multinomial regression analysis were conducted.

\section{Results}

\section{Demographic Characteristics}

Of the 1050 total subjects, 487 (46.4\%) were female. Their mean age was 44.6 years ( $\pm 10.2 \mathrm{SD}$; range, 19 to 80 years). The proportions of subjects with hypertension, diabetes, and obesity were $9.3 \%, 2.5 \%$, and $29.5 \%$, respectively. Following the Rome III criteria, the prevalence of IBS, functional constipation, functional diarrhea, and FD were $11.5 \%, 11.4 \%, 7.5 \%$, and $9.7 \%$, respectively.

\section{Prevalence and Clinical Characteristics of Abdominal Bloating}

The prevalence of abdominal bloating was $26.9 \%$ (282 of 1050 subjects) with a female predominance ( $52.5 \%$ vs $44.1 \%, P=0.016)$ (Table 1). The mean age of subjects who experienced bloating was lower than that of non-bloating controls (42.7 vs 45.3 years), with a significant decreasing trend with increasing age $(P<0.001)$ (Figure). The proportion of subjects younger than 40 years with bloating was significantly higher than that in the older group $(34.5 \%$ vs $23.1 \%$, $P<0.001)$. Bloating was detected more frequently among female than male subjects $(30.4 \%$ vs $23.8 \%, P=0.016)$. However, the proportion of obese subjects, having comorbidities including hypertension, diabetes mellitus, stroke, cholecystectomy, or smoking habit was not different between the bloating group and controls. FGIDs were detected more frequently in the bloating group than in the controls. The proportion of subjects with bloating was $72.7 \%$ among subjects with IBS and $60.8 \%$ among subjects with functional constipation.

\section{Prevalence of Functional Abdominal Bloating}

After exclusion of other overlapping FGIDs, the prevalence of functional bloating by the Rome III criteria was $6.9 \%$.

In multivariate analysis, using multiple logistic regression, the presence of abdominal bloating was associated with younger age less than 40 years $(\mathrm{OR}, 2.03 ; 95 \% \mathrm{CI}, 1.34-3.08 ; P=0.001)$ and a high somatization score (OR, 4.58; 95\% CI, 2.76-7.58; $P<0.001)$ (Table 2).

\section{Factor Analysis of Symptom Groupings}

Principal component analysis of the dataset (factor analysis) using the Kaiser criterion revealed the presence of a 5-factor structure of the symptoms when the intensity grading of each GI symptom 
Table 1. Demographics and Clinical Factors Associated with Abdominal Bloating

\begin{tabular}{|c|c|c|c|}
\hline Variable & $\begin{array}{l}\text { Bloating } \\
(\mathrm{n}=282)\end{array}$ & $\begin{array}{l}\text { Controls } \\
(\mathrm{n}=768)\end{array}$ & $P$-value \\
\hline Mean age $\pm \mathrm{SD}(\mathrm{yr})$ & $42.7 \pm 10.6$ & $45.3 \pm 9.9$ & $<0.001$ \\
\hline Age below $40 \mathrm{yr}$ & $119(42.3)$ & $226(29.4)$ & $<0.001$ \\
\hline Female gender & $148(52.5)$ & $339(44.1)$ & 0.016 \\
\hline $\mathrm{BMI}>25 \mathrm{~kg} / \mathrm{m}^{2}$ & $83(29.4)$ & $227(29.6)$ & 0.969 \\
\hline \multicolumn{4}{|l|}{ Underlying diseases } \\
\hline Hypertension $(\mathrm{n}=674)$ & $21(11.3)$ & $77(15.8)$ & 0.140 \\
\hline Diabetes mellitus $(\mathrm{n}=674)$ & $7(3.8)$ & $19(3.9)$ & 0.938 \\
\hline Stroke $(\mathrm{n}=674)$ & $0(0.0)$ & $5(1.0)$ & 0.330 \\
\hline Cholecystectomy & $3(1.1)$ & $8(1.0)$ & 1.000 \\
\hline High Somatization score $(\mathrm{n}=1044)$ & $90(32.3)$ & $79(10.3)$ & $<0.001$ \\
\hline Self-reported depressed mood & $155(55.0)$ & $254(33.1)$ & $<0.001$ \\
\hline Stool consistency ${ }^{\mathrm{a}}$ & & & $<0.001$ \\
\hline Hard stool (Bristol scale 1-2) & $19(6.7)$ & $25(3.3)$ & \\
\hline Normal stool (Bristol 3-5) & $196(69.5)$ & $648(84.4)$ & \\
\hline Watery stool (Bristol 6-7) & $67(23.8)$ & $95(12.4)$ & \\
\hline Stool frequency (times per week) & $7.0 \pm 4.1$ & $7.5 \pm 3.4$ & 0.063 \\
\hline \multicolumn{4}{|l|}{ Functional GI disorders } \\
\hline Functional dyspepsia $(\mathrm{n}=102)$ & $67(23.8)$ & $35(4.6)$ & $<0.001$ \\
\hline Irritable bowel syndrome $(\mathrm{n}=121)$ & $88(31.2)$ & $33(4.3)$ & $<0.001$ \\
\hline Functional constipation $(\mathrm{n}=120)$ & $73(25.9)$ & $47(6.1)$ & $<0.001$ \\
\hline Functional diarrhea $(\mathrm{n}=79)$ & $43(15.2)$ & $36(4.7)$ & $<0.001$ \\
\hline Current smoking $(\mathrm{n}=661)$ & $44(24.2)$ & $98(20.5)$ & 0.299 \\
\hline Alcohol user $(\mathrm{n}=641)$ & $119(67.6)$ & $305(65.6)$ & 0.629 \\
\hline Lack of exercise $(\mathrm{n}=651)$ & $72(39.8)$ & $143(30.4)$ & 0.023 \\
\hline
\end{tabular}

${ }^{a}$ Bristol stool form scale. ${ }^{15}$

SD, standard deviation; BMI, body mass index; GI, gastrointestinal.

$P$-value $<0.05$, significant. $P$-values were calculated using $t$ test (for continuous variables) or $\chi^{2}$ test (categorical variables).

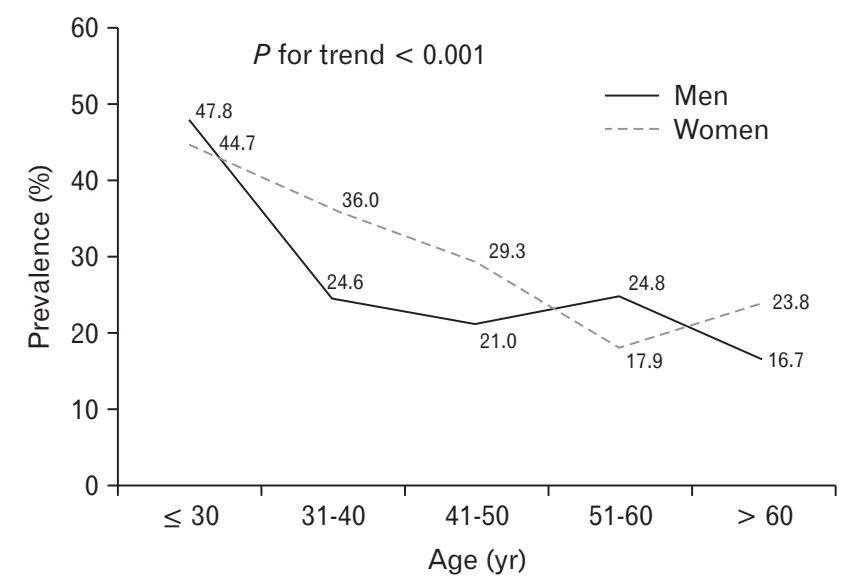

Figure. Prevalence of abdominal bloating according to age and gender. The proportion of bloating is significant decreasing by aging. score was used (Table 3). Factor analysis revealed that 19 items could be categorized into 5 symptom factors. These 5 factors explained $62.3 \%$ of the variance, with factors $1,2,3,4$, and 5 accounting for $29.4 \%, 11.0 \%, 9.2 \%, 6.8 \%$, and $5.9 \%$, respectively, of the total variance.

The factor loadings of the items exceeded 0.4 (Table 3). These 5 factors could be broadly interpreted as follows. Factor 1 comprised early satiety, epigastric pain, heartburn, postprandial fullness, abdominal pain, acid regurgitation, and abdominal bloating. This factor was related to upper GI symptoms, consisting of FD and functional heartburn by the Rome III criteria. Factor 2 included anorectal obstruction, straining, incomplete evacuation, hard stool and manual maneuver. These symptoms were related to functional constipation, especially defecatory disorder of constipation. Factor 3 comprised frequent bowel movements, more loose stools, abdominal pain relieved by defecation and diarrhea which are related to diarrhea-predominant IBS (D-IBS) by the Rome III criteria. Fac- 
Table 2. Logistic Analysis of Associated Factors for Abdominal Bloating

\begin{tabular}{|c|c|c|c|c|c|c|}
\hline & \multicolumn{3}{|c|}{ Univariate analysis } & \multicolumn{3}{|c|}{ Multivariate analysis } \\
\hline & OR & $95 \% \mathrm{CI}$ & $P$-value & OR & $95 \% \mathrm{CI}$ & $P$-value \\
\hline Age below 40 yr & 1.75 & $1.32-2.32$ & $<0.001$ & 2.03 & $1.34-3.08$ & 0.001 \\
\hline Female gender & 1.40 & $1.06-1.84$ & 0.016 & 0.94 & $0.60-1.48$ & 0.779 \\
\hline Hypertension & 0.68 & $0.41-1.14$ & 0.141 & 0.76 & $0.41-1.40$ & 0.374 \\
\hline Diabetes mellitus & 0.97 & $0.40-2.34$ & 0.938 & 1.10 & $0.38-3.13$ & 0.866 \\
\hline High somatization & 4.14 & $2.94-5.82$ & $<0.001$ & 4.58 & $2.76-7.58$ & $<0.001$ \\
\hline Depression & 2.47 & $1.87-3.26$ & $<0.001$ & 1.30 & $0.85-2.01$ & 0.231 \\
\hline Current smoking & 1.24 & $0.83-1.86$ & 0.299 & 1.20 & $0.73-1.95$ & 0.471 \\
\hline Alcohol user & 1.10 & $0.76-1.58$ & 0.629 & 1.17 & $0.75-1.82$ & 0.491 \\
\hline Lack of exercise & 1.51 & $1.06-2.16$ & 0.023 & 1.14 & $0.75-1.74$ & 0.539 \\
\hline
\end{tabular}

OR, odds ratio; $\mathrm{CI}$, confidence interval.

Adjusted ORs were obtained by means of multivariable logistic regression analysis with covariates that were regarded as associating abdominal bloating.

Table 3. Factor Loading of Gastrointestinal Symptoms in Factor Analysis

\begin{tabular}{|c|c|c|c|c|c|}
\hline Factor & 1 & 2 & 3 & 4 & 5 \\
\hline Early satiety & 0.81 & 0.12 & 0.07 & 0.12 & -0.03 \\
\hline Epigastric pain & 0.79 & 0.07 & 0.12 & 0.02 & 0.01 \\
\hline Heartburn & 0.67 & 0.16 & -0.03 & 0.10 & 0.12 \\
\hline Postprandial fullness & 0.66 & 0.08 & 0.36 & 0.30 & -0.09 \\
\hline Abdominal pain & 0.65 & 0.08 & 0.09 & -0.02 & 0.04 \\
\hline Acid regurgitation & 0.51 & 0.15 & 0.14 & -0.02 & 0.28 \\
\hline Bloating & 0.51 & 0.40 & 0.28 & 0.17 & -0.07 \\
\hline Anorectal obstruction & 0.21 & 0.84 & 0.12 & 0.12 & 0.02 \\
\hline Straining & 0.14 & 0.81 & 0.14 & 0.18 & 0.00 \\
\hline Incomplete evacuation & 0.26 & 0.72 & 0.22 & 0.07 & 0.12 \\
\hline Hard stool & 0.10 & 0.68 & 0.05 & 0.24 & 0.02 \\
\hline Manual maneuver & 0.01 & 0.50 & -0.09 & -0.02 & -0.01 \\
\hline Frequent bowel movement & 0.10 & 0.04 & 0.86 & 0.05 & 0.00 \\
\hline More loose stool & 0.13 & 0.09 & 0.83 & 0.01 & 0.18 \\
\hline Pain relieved by defecation & 0.19 & 0.05 & 0.70 & 0.30 & -0.18 \\
\hline Diarrhea & 0.25 & 0.24 & 0.49 & -0.22 & 0.38 \\
\hline Harder stool & 0.07 & 0.22 & 0.08 & 0.82 & 0.04 \\
\hline Rare bowel movement & 0.16 & 0.19 & 0.09 & 0.75 & 0.12 \\
\hline Fecal incontinence & 0.08 & -0.00 & 0.03 & 0.17 & 0.89 \\
\hline
\end{tabular}

Extraction method, principle component analysis; Rotation method, varimax with Kaiser normalization.

Bold values indicate the significance of highest loading values for each factor.

Factor 1 was related to upper gastrointestinal symptoms, consisting of functional dyspepsia and functional heartburn. Factor 2 was related to functional constipation, especially defecatory disorder of constipation. Factor 3 was related to diarrhea-predominant irritable bowel syndrome (IBS). Factor 4 was related to constipationpredominant IBS. Factor 5 indicated fecal incontinence.

tor 4 included harder stools and rare bowel movements, suggestive of constipation-predominant IBS (C-IBS); fecal incontinence was an independent factor as factor 5 . In the present study, the loading factor of bloating, which represented the correlations between the common factor and the input variables, was 0.51 for factor 1 and 0.40 for factor 2. Therefore, bloating loaded marginally on both factors 1 and 2 .

\section{Diagnostic Role of Abdominal Bloating for Functional Gastrointestinal Disorders}

There were significant overlaps between abdominal bloating and several FGIDs. Abdominal bloating was highly prevalent in FD and IBS (Table 4). The unadjusted OR of abdominal bloating was 6.5 for FD only $(P<0.001)$ and 10.1 for IBS $(P<0.001)$. 
Table 4. Impacts of Abdominal Bloating on Functional Gastrointestinal Disorders by Multinomial Logistic Analysis

\begin{tabular}{|c|c|c|c|c|c|c|c|}
\hline \multirow{3}{*}{ Total $(\mathrm{N}=1050)$} & \multirow{3}{*}{$\begin{array}{l}\text { Bloating } \\
(\mathrm{n}=282)\end{array}$} & \multicolumn{2}{|c|}{ Unadjusted } & \multicolumn{4}{|c|}{ Adjusted } \\
\hline & & \multirow{2}{*}{ OR } & \multirow{2}{*}{$P$-value } & \multicolumn{2}{|c|}{ Model $^{\mathrm{a}}$} & \multicolumn{2}{|c|}{ Model $2^{b}$} \\
\hline & & & & OR & $P$-value & OR & $P$-value \\
\hline $\operatorname{IBS}(\mathrm{n}=121, \mathrm{n}[\%])$ & $88(72.7)$ & 10.1 & $<0.001$ & 8.6 & $<0.001$ & 7.5 & $<0.001$ \\
\hline C-IBS $(n=14)$ & $13(92.9)$ & 37.1 & $<0.001$ & 43.8 & $<0.001$ & 40.0 & $<0.001$ \\
\hline D-IBS $(n=63)$ & $47(74.6)$ & 9.4 & $<0.001$ & 8.0 & $<0.001$ & 7.0 & $<0.001$ \\
\hline M-IBS $(\mathrm{n}=25)$ & $16(64.0)$ & 5.1 & $<0.001$ & 3.3 & 0.008 & 3.3 & 0.012 \\
\hline U-IBS $(\mathrm{n}=19)$ & $12(63.2)$ & 4.8 & $<0.001$ & 4.8 & 0.002 & 3.7 & 0.012 \\
\hline $\mathrm{FD}(\mathrm{n}=102, \mathrm{n}[\%])$ & $67(65.7)$ & 6.5 & $<0.001$ & 4.7 & $<0.001$ & 3.7 & 0.002 \\
\hline EPS only $(n=40)$ & $21(52.5)$ & 3.2 & $<0.001$ & 2.0 & $<0.001$ & 1.5 & $<0.001$ \\
\hline PDS only $(\mathrm{n}=31)$ & $21(67.7)$ & 6.1 & $<0.001$ & 4.7 & $<0.001$ & 4.7 & $<0.001$ \\
\hline Overlap of PDS/EPS $(n=31)$ & $25(80.6)$ & 12.4 & $<0.001$ & 9.6 & $<0.001$ & 7.0 & $<0.001$ \\
\hline
\end{tabular}

${ }^{a}$ Multinomial logistic analysis adjusted by age, sex and somatization symptom score.

${ }^{\mathrm{b}}$ Multinomial logistic analysis adjusted by age, sex, somatization symptom score, and other gastrointestinal symptoms which were not constituted with functional dyspepsia (FD) and irritable bowel syndrome (IBS).

C-IBS, constipation-predominant IBS; D-IBS, diarrhea-predominant IBS; M-IBS, mixed IBS; U-IBS, unsubtyped IBS; EPS, epigastric pain syndrome; PDS, postprandial distress syndrome.

After adjustment for age, gender, and somatization symptom score, the OR for bloating was 4.7 for FD and 8.6 for IBS (Model 1). In Model 2, other GI symptoms that were not involved in FD or IBS were added to the multinominal logistic analysis. The OR for bloating was 3.7 for $\operatorname{FD}(P=0.002)$ and 7.5 for IBS $(P<0.001)$. According to the subtype of $\mathrm{FD}$, bloating was revealed to be associated with more increased OR for postprandial distress syndrome than that for epigastric pain syndrome. C-IBS was the most related subtype of IBS with the presence of bloating (OR, 40.0; $P<0.001)$.

According to the severity of IBS, bloating was noted in $67.8 \%$ of mild IBS, $73.5 \%$ of moderate IBS, and $84.6 \%$ of severe IBS $(P$ $<0.001)$. However, there was no significant difference in the presence of bloating according to the severity of FD.

\section{Discussion}

Abdominal bloating is common in the general population, and people often report concomitant occurrence of multiple symptoms of FGIDs. We documented the prevalence of abdominal bloating and its strong association with FGID symptom complexes in a large health-screening cohort. Abdominal bloating was present in $26.9 \%$ of the study population, and functional bloating by the Rome III criteria was present in $6.9 \%$ of the subjects. This result is consistent with a previous Western population study, and higher than that of an Asian population report. ${ }^{6,16,17}$ Epidemiologic studies have revealed that $15-30 \%$ of the general United States (US) population experiences bloating symptoms, compared with about $15 \%$ reported in an Asian population.,

The present study revealed that abdominal bloating was more strongly associated with IBS than FD and bloating might be an independent predictor of IBS severity. In previous studies, the prevalence of bloating ranged from $66 \%$ to $90 \%$ among patients with IBS, and bloating has been correlated with IBS according to the severity. $^{6,717-19}$ In a US telephone survey of lower GI symptoms, more than $74-77 \%$ of subjects considered their symptoms as moderateto-severe, and nearly $10-15 \%$ reported that their daily activities were reduced by $50 \%$ or more. ${ }^{6}$ However, in Asian clinical practice, bloating is sometimes considered as the suffering of symptoms originating from the stomach, and many Malays seem to complain of "a lot of wind" in the stomach. ${ }^{10}$ Several factor analyses of upper GI symptoms revealed that bloating was grouped into other upper GI symptoms. ${ }^{11,20}$ In the largest face-to face survey conducted in Europe, factor analysis revealed that dyspepsia symptoms were underscored when using a 3-factor structure that included bloating. ${ }^{11}$ In a telephone survey conducted in a large US general population, bloating was categorized into a unique cluster including pain/discomfort in the abdomen, and cluster analysis confirmed bloating as an independent cluster, different from the fullness/early satiation, gastroesophageal reflux disease, and nausea/vomiting clusters. However, this study evaluated mainly upper GI symptoms and did not include lower GI symptoms. However, the results of factor analysis could be altered according to the symptom items selected. When we performed factor analysis involving 19 upper and lower GI symptoms, abdominal bloating was loaded on both the upper 
GI symptoms and constipation groups. However, when a factor analysis with only 7 upper GI symptoms was performed, bloating was highly correlated with FD symptoms (loading factor 0.81, data not published). Therefore, the groupings of GI symptoms identified by factor analysis are dependent on the subjects and content of the survey questions. As compared with previous surveys, our questionnaire included more comprehensive questions on upper and lower GI symptoms.

IBS is a common medical condition, but includes heterogeneous symptoms. Some physicians believe that IBS is a distinct FGID defined using a biopsychosocial model, but others believe that IBS, as a diagnostic entity, will disappear when its organic causes are identified. Making a positive diagnosis of IBS has important implications in terms of reassuring the patient, and it may minimize usage of medical resources and direct treatment in a logical manner. The Manning criteria included abdominal pain relieved by defecation, more frequent or loose stools with onset of pain, feeling of incomplete emptying, passage of mucus via the rectum or visible abdominal distension. ${ }^{21}$ The Rome I criteria involve similar symptoms as the Manning criteria, except with the additional consideration of symptom duration. ${ }^{22}$ However, in the Rome II symptom criteria, bloating was excluded. In factor analysis, the bloating symptom was covaried with IBS symptoms in only male subjects. ${ }^{23}$ Three Rome II core symptoms (abdominal pain and pain relieved by defecation or bowel habit changes) were clustered together in US, Australian, German, and Swedish community surveys. ${ }^{24}$ In these studies, abdominal pain was reported according to different abdominal locations; bloating was weakly covaried with the constipation and diarrhea groups, but not lower or upper abdominal pain. The difference between the Rome II and III IBS definitions is the symptom onset and duration of the IBS symptom complex. ${ }^{25}$ However, there are limited data on the accuracy of the Rome III criteria in differentiating IBS from organic disease in the era of impeding Rome IV criteria. In the present study, abdominal bloating was weakly clustered with individual symptom items; therefore, we performed a multinomial logistic analysis to evaluate the impact of abdominal bloating on the IBS symptom cluster. In the present study, the presence of abdominal bloating was increased by 7.5 -fold for IBS and by 40.0 -fold for C-IBS.

In 2012, an international IBS experts' meeting indicated the need for revised criteria for the diagnosis of IBS; in particular, inclusion of bloating was considered critical. ${ }^{26}$ This survey reported that bloating was stated by over half of the experts to be the most important feature of IBS, and only one quarter of the experts felt abdominal pain to be the most bothersome symptom. Two studies have suggested abdominal bloating to be one of the most bothersome symptoms in IBS. ${ }^{7,8}$ Also, bloating symptoms are associated with a low level of health-related quality of life, especially physical functioning, including lower energy and food intake, anxiety, and depression. ${ }^{8,27,28}$ There are few data on the use of medications for bloating symptoms in patients with IBS. In a study on tegaserod in IBS, bloating was the second most common symptom for indication of prescribing this medication (80\%), following abdominal pain or discomfort (87\%). ${ }^{29}$ In a survey of experts, normalized stool form/ frequency was selected by $76.9 \%$ of participants, while $61.5 \%$ suggested improvements in pain and $48.7 \%$ improvements in bloating. ${ }^{26}$

The appropriate outcome measures for IBS are one of the most important issues in the development of a drug to resume normal bowel functioning, and the Food and Drug Administration guidelines recommend that C-IBS and D-IBS should be studied in separate clinical trials because the clinical sign and symptoms are significantly different. ${ }^{30}$ Therefore, bloating might be an appropriate secondary outcome of IBS, especially C-IBS, in clinical trials.

A major limitation of this study lies in the evaluation of the accuracy of bloating for diagnosing IBS, namely, the lack of a reference standard for this disorder. The study population included subjects undergoing a medical check-up, and one-third of subjects underwent colonoscopy, which can exclude organic lower GI diseases such as diverticulitis, inflammatory bowel disease, and colon cancer. However, all study subjects underwent the stool occult blood test, blood cell counts, blood chemistry evaluation, thyroid functional test, abdomen ultrasonography or upper GI evaluation, including upper endoscopy or a barium study, and reporting of medical history. Therefore, other organic diseases that provoke upper and lower GI symptoms would likely have been excluded. Also, we assessed IBS severity by only frequency of abdominal pains only, and not including the quality of life. Finally, we did not perform a physiologic assessment to evaluate the pathogenesis of bloating with/without distension. Further studies on the pathogenesis and/or psychosocial dimension of bloating are thus necessary.

In conclusion, abdominal bloating is common and frequently included with FGIDs, but bloating is similarly correlated with upper and lower GI symptoms. However, abdominal bloating is a more predictable for IBS, especially in C-IBS, than FD. Also bloating is more frequently combined with IBS according to their symptom severity. Therefore, bloating might be an appropriate secondary outcome of IBS, especially C-IBS, in clinical trials. 


\section{Financial support: None.}

\section{Conflict of interest: None.}

Author contributions: Min Sun Ryu is the guarantor of the article and contributed to data analysis and critical revision of manuscript; Hye-Kyung Jung contributed to study supervision, study design, data analysis, drafting of manuscript, and critical revision of manuscript; Jae-In Ryu was involved in data analysis and drafting of manuscript; Jung-Sook Kim contributed to acquisition of data; and Kyung Ae Kong was involved in data analysis.

\section{References}

1. Schmulson M, Chang L. Review article: the treatment of functional abdominal bloating and distension. Aliment Pharmacol Ther 2011;33: 1071-1086.

2. Houghton LA, Whorwell PJ. Towards a better understanding of abdominal bloating and distension in functional gastrointestinal disorders. Neurogastroenterol Motil 2005;17:500-511.

3. Talley NJ, Zinsmeister AR, Van Dyke C, Melton LJ 3rd. Epidemiology of colonic symptoms and the irritable bowel syndrome. Gastroenterology 1991;101:927-934.

4. Sandler RS, Drossman DA, Nathan HP, McKee DC. Symptom complaints and health care seeking behavior in subjects with bowel dysfunction. Gastroenterology 1984;87:314-318.

5. Drossman DA, McKee DC, Sandler RS, et al. Psychosocial factors in the irritable bowel syndrome. A multivariate study of patients and nonpatients with irritable bowel syndrome. Gastroenterology 1988;95:701-708.

6. Sandler RS, Stewart WF, Liberman JN, Ricci JA, Zorich NL. Abdominal pain, bloating, and diarrhea in the United States: prevalence and impact. Dig Dis Sci 2000;45:1166-1171.

7. Lembo T, Naliboff B, Munakata J, et al. Symptoms and visceral perception in patients with pain-predominant irritable bowel syndrome. Am J Gastroenterol 1999;94:1320-1326.

8. Ringel Y, Williams RE, Kalilani L, Cook SF. Prevalence, characteristics, and impact of bloating symptoms in patients with irritable bowel syndrome. Clin Gastroenterol Hepatol 2009;7:68-72.

9. Ho KY, Kang JY, Seow A. Prevalence of gastrointestinal symptoms in a multiracial Asian population, with particular reference to reflux-type symptoms. Am J Gastroenterol 1998;93:1816-1822.

10. Goh KL. Clinical and epidemiological perspectives of dyspepsia in a multiracial Malaysian population. J Gastroenterol Hepatol 2011;26(suppl 3): 35-38.

11. Piessevaux H, De Winter B, Louis E, et al. Dyspeptic symptoms in the general population: a factor and cluster analysis of symptom groupings. Neurogastroenterol Motil 2009;21:378-388.

12. Tack J, Piessevaux H, Coulie B, Caenepeel P, Janssens J. Role of impaired gastric accommodation to a meal in functional dyspepsia. Gastroenterology 1998;115:1346-1352.

13. Song HJ, Jung HK. Reliability and validity of Korean bowel disease questionnaire for functional gastrointestinal disorders. Ewha Med J
2011;34:39-46.

14. Rome Foundation. Guidelines--Rome III diagnostic criteria for functional gastrointestinal disorders. J Gastrointestin Liver Dis 2006;15:307-312.

15. Lewis SJ, Heaton KW. Stool form scale as a useful guide to intestinal transit time. Scand J Gastroenterol 1997;32:920-924.

16. Jiang X, Locke GR 3rd, Choung RS, Zinsmeister AR, Schleck CD, Talley NJ. Prevalence and risk factors for abdominal bloating and visible distention: a population-based study. Gut 2008;57:756-763.

17. Drossman DA, Li Z, Andruzzi E, et al. U.S. householder survey of functional gastrointestinal disorders. Prevalence, sociodemography, and health impact. Dig Dis Sci 1993;38:1569-1580.

18. Drossman DA, Morris CB, Schneck S, et al. International survey of patients with IBS: symptom features and their severity, health status, treatments, and risk taking to achieve clinical benefit. J Clin Gastroenterol 2009;43:541-550.

19. Spiegel B, Strickland A, Naliboff BD, Mayer EA, Change L. Predictors of patient-assessed illness severity in irritable bowel syndrome. Am J Gastroenterol 2008;103:2536-2543.

20. Camilleri M, Dubois D, Coulie B, et al. Prevalence and socioeconomic impact of upper gastrointestinal disorders in the United States: results of the US Upper Gastrointestinal Study. Clin Gastroenterol Hepatol 2005;3:543-552

21. Manning AP, Thompson WG, Heaton KW, Morris AF. Towards positive diagnosis of the irritable bowel. Br Med J 1978;2:653-654.

22. Drossman DA, Thompson WG, Talley NJ, Funch-Jensen P, Janssens J, Whitehead WE. Identification of sub-groups of functional gastrointestinal disorders. Gastroenterology Int 1990;3:159-172.

23. Taub E, Cuevas JL, Cook EW 3rd, Crowell M, Whitehead WE. Irritable bowel syndrome defined by factor analysis. Gender and race comparisons. Dig Dis Sci 1995;40:2647-2655.

24. Talley NJ, Holtmann G, Agréus L, Jones M. Gastrointestinal symptoms and subjects cluster into distinct upper and lower groupings in the community: a four nations study. Am J Gastroenterol 2000;95:1439-1447.

25. Longstreth GF, Thompson WG, Chey WD, Houghton LA, Mearin F, Spiller RC. Functional bowel disorders. Gastroenterology 2006;130:1480-1491.

26. Pimentel M, Talley NJ, Quigley EM, Hani A, Sharara A, Mahachai V. Report from the multinational irritable bowel syndrome initiative 2012. Gastroenterology 2013;144:e1-e5.

27. Chang L, Lee OY, Naliboff B, Schmulson M, Mayer EA. Sensation of bloating and visible abdominal distension in patients with irritable bowel syndrome. Am J Gastroenterol 2001;96:3341-3347.

28. Jung HK, Kim YH, Park JY, et al. Estimating the burden of irritable bowel syndrome: analysis of a nationwide Korean database. J Neurogastroenterol Motil 2014;20:242-252.

29. Bradette M, Wawer AR, Balshaw R, Kelly S, Barbeau M, Sambrook R. Characteristics, diagnostic and symptom profile of patients receiving tegaserod in routine clinical practice in Canada. Can J Clin Pharmacol 2007; 14:e291-e300.

30. Guidance for industry irritable bowel syndrome — clinical evaluation of drugs for treatment. Available from URL: http://www.fda.gov/downloads/Drugs/.../Guidances/UCM205269.pdf (accessed 20 Sep 2015). 